\title{
DISK OSCILLATIONS AS THE ORIGIN OF QUASI-PERIODIC OSCILLATIONS IN BLACK HOLE CANDIDATES
}

\author{
T. YAMASAKI \\ Hida Observatory, Kyoto University \\ Kamitakara, Gifu 506-13 \\ AND \\ S. MINESHIGE AND S. KATO \\ Department of Astronomy, Faculty of Science, Kyoto University \\ Sakyo-ku, Kyoto 606-01
}

Quasi-periodic oscillations (QPOs) of a few $\mathrm{Hz}$ are observed in the very high state of some black hole candidates (GX 339-4 and GS 1124-68). This is the Kepler frequency at the radius of a few hundred Schwarzschild radii. As a possible mechanism of the QPOs in these objects, the trapped oscillations in the accretion disks are considered. The trapped oscillations of the disks were investigated by several authors. They studied the trapped oscillations in the standard radiative cooling-dominated disks. Recently, the advection-dominated accretion flow is considered, as a possible model to explain the hard X-ray spectra of the black hole candidates or the active galactic nuclei. In particular, in the very high state of some black hole candidates, the spectrum can be explained by the disk-corona model which comprises the cold standard accretion disk and the advection-dominated corona above the cold disk. We thus investigated the trapped axi-symmetric oscillations in the advection-dominated corona by the global linear analysis.

The results show that, i)the periods of the trapped oscillations are $0.1 \Omega_{\text {out }} \sim 2 \Omega_{\text {out }}$. Here $\Omega_{\text {out }}$ is the Kepler frequency in the outer boundary. In the case of the black hole of $10 M_{\odot}$, the Kepler frequency is about $7 \mathrm{~s}^{-1}$ at the radius of 100 Schwarzschild radii. ii)As the advective cooling becomes more efficient, the frequencies become large. iii)Owing to the radial inflow in the unperturbed flow, the phase of the oscillation differs with radius. Because of this, the shape of the light curve deviate from the sinusoidal one. 